\title{
Prevalence and correlates of medication non-adherence among kidney transplant recipients more than 6 months post-transplant: a cross-sectional study
}

Francis $L$ Weng ${ }^{1,2^{*}}$, Sheenu Chandwani ${ }^{2}$, Karen M Kurtyka ${ }^{2}$, Christopher Zacker ${ }^{3}$, Marie A Chisholm-Burns ${ }^{4}$ and Kitaw Demissie ${ }^{2}$

\begin{abstract}
Background: Among kidney transplant recipients, non-adherence with immunosuppressive medications frequently precedes allograft loss. We sought to determine the prevalence and correlates of medication non-adherence among kidney transplant recipients.

Methods: We performed a single-center, cross-sectional study of kidney transplant recipients who were at least 6 months post-transplant. We measured self-reported adherence using the Immunosuppressive Therapy Adherence Scale (ITAS, which is scored from 0 to 12, where higher scores indicate increased adherence) and barriers to adherence using the Immunosuppressive Therapy Barriers Scale (ITBS). We also used validated scales to measure perceived stress, health literacy, anxiety, depression, and interpersonal support.

Results: The 252 patients included in the study were $59.9 \%$ male, 27.0\% Black, and at a median of 2.9 years post-transplant (interquartile range [IQR] 1.4-5.8). On the ITAS, 59.1\% scored a perfect 12, 26.6\% scored 10-11, and 14.3\% scored 0-9. In univariate models, non-adherence (defined as ITAS score $\leq 9$ ) was significantly associated with increased scores on scales for perceived stress (OR 1.12, 95\% Cl 1.01-1.25) and depression (OR 1.14, 95\% Cl 1.02-1.28), and with more self-reported barriers to adherence on the ITBS (OR 1.15, 95\% Cl 1.08-1.22). After adjusting for sociodemographic factors, stress and depression were not associated with non-adherence. Higher scores on the ITBS (corresponding to more self-described barriers to adherence) were associated with lower scores on the ITAS $(\mathrm{P}<0.001)$. Several individual barriers were associated with non-adherence.
\end{abstract}

Conclusions: Among prevalent kidney transplant recipients, a minority is non-adherent. Practical barriers to adherence may serve as promising targets for future interventions.

Keywords: Kidney transplantation, Epidemiology, Compliance, Adherence

\section{Background}

Among recipients of kidney transplants, non-adherence with prescribed immunosuppressive medications commonly occurs and frequently precedes allograft loss [1-3]. A recent systematic review reported that in fifteen cross-sectional studies, a median of $22.3 \%$ of kidney

\footnotetext{
* Correspondence: fweng@barnabashealth.org

'Saint Barnabas Medical Center, Renal \& Pancreas Transplant Division, 94 Old Short Hills Road, East Wing, Suite 305, Livingston, New Jersey 07039, USA ${ }^{2}$ Department of Epidemiology, Rutgers School of Public Health, 683 Hoes Lane West, Piscataway, New Jersey 08854, USA

Full list of author information is available at the end of the article
}

transplant recipients were non-adherent [1]. Furthermore, in ten cohort studies, a median of $36.4 \%$ of kidney allograft losses were associated with prior non-adherence [1]. Compared to recipients of other solid organ transplants, kidney transplant recipients may demonstrate higher rates of non-adherence [4].

Several factors are associated with post-transplant medication non-adherence [5]. Patient-related factors associated with non-adherence include younger age [2,6,7], increased time since the transplant [6], and possibly Black race [8]. Possible barriers to adherence include patients'

\section{Biomed Central}


personal schedules and routines [9], characteristics of the medicines and their dosage and schedules [8,9], practical issues related to access to medications and pharmacy refills [9], and medication costs [10]. Provider- and health systems-related factors also may contribute significantly to post-transplant medication adherence [4]. Unfortunately, few trials have tested interventions designed to increase adherence among transplant recipients [11]. Development of effective adherence interventions for transplant recipients requires a better understanding of factors potentially associated with non-adherence.

In this study, we sought to determine, among a population of stable, adult kidney transplant recipients who were more than 6 months post-transplant, (1) the prevalence of self-reported medication non-adherence, (2) psychosocial correlates of non-adherence, and (3) selfreported barriers to medication adherence.

\section{Methods}

\section{Study design}

A cross-sectional study was conducted in the outpatient transplant clinic of the Renal and Pancreas Transplant Division at Saint Barnabas Medical Center in Livingston, New Jersey, USA. To be eligible for this study, patients had to (1) have a functioning kidney-only transplant; (2) have received the transplant at Saint Barnabas Medical Center; (3) be at least 6 months post-transplant; (4) be 18 years of age or older at the time of the study; (5) be able to understand English; and (6) give informed consent. We excluded patients who had received a non-renal solid organ transplant, a simultaneous pancreas-kidney transplant, or more than one kidney transplant. The study was approved by the human subjects Institutional Review Boards at both Saint Barnabas Medical Center and the University of Medicine and Dentistry of New Jersey.

\section{Study procedures}

We enrolled kidney transplant recipients who came for an appointment in our outpatient transplant clinic between May and September 2011. Prior to each clinic session, we identified the scheduled patients who met the study eligibility criteria. During the clinic visit, the nephrologist described the study to patients and invited them to participate. Interested patients then met with study personnel to confirm study eligibility, provide informed consent, and complete the study's self-administered questionnaires. Study instruments were all completed in-person at the time of the clinic and study visit. After completing the questionnaires, study participants were given a $\$ 15$ gift card in appreciation of their participation.

\section{Self-report instruments}

Adherence was measured using the self-administered Immunosuppressive Therapy Adherence Scale (ITAS).
The ITAS is a 4-item, 12-point scale that has been validated in kidney transplant recipients [12] and used in other studies of medication adherence [13,14]. A higher score corresponds with increased adherence.

Barriers to adherence were measured using the Immunosuppressive Therapy Barriers Scale (ITBS), a reliable and validated scale (Cronbach's alpha 0.91) [15]. The ITBS is a 13-item scale consisting of 5-point Likert responses that rate self-reported agreement with eight "uncontrollable" factors and five "controllable" factors. Scores range from 13 to 65 . A higher score corresponds with more barriers to adherence.

Participants also completed self-report symptom rating scale, including Hospital Anxiety and Depression Scale (HADS) [16], Interpersonal Support and Evaluation List-12 (ISEL-12) [17,18], and Perceived Stress Scale-4 (PSS-4) $[19,20]$. On the HADS, anxiety and depression are each measured on a 22-point scale from 0 to 21, with a higher score denoting more anxiety or depression. Scale subscores for anxiety and depression of $0-7,8-10$, and $11-21$ corresponded with no, doubtful, or definite anxiety or depression, respectively [16]. On the ISEL-12, social support is scored from 12 to 48, with a higher score corresponding to higher social support. On the PSS-4, perceived stress is rated from 0 to 16, with a higher score correlating to increased perceived stress. There are no scores cut-offs for the ISEL-12 or PSS-4.

Participants also completed the Short Test of Functional Health Literacy in Adults (sTOFHLA) [21]. On the sTOFHLA, health literacy is measured from 0 to 36 , with a score of $0-16$ deemed as "inadequate", 17-22 as "marginal", and 23-36 as "adequate" literacy.

\section{Statistical analysis}

Categorical variables were summarized as proportions, and their estimates across groups (e.g. adherence categories) were compared using chi-square testing or Fisher's exact test as appropriate. Continuous variables that were not normally distributed were summarized as medians with 25\%-75\% interquartile ranges (IQRs) and compared using Wilcoxon rank-sum tests. We used binary logistic regression [22] to model the unadjusted associations between independent variables (psychosocial and demographic covariates) and self-reported non-adherence, with non-adherence defined as an ITAS score of 9 or less. The Likert responses on the ITBS were modeled as ordinal variables. Candidate variables with $\mathrm{P}<0.20$ in the univariate analysis were eligible for inclusion in the multivariate models [22]. For the multivariate models, we adjusted each candidate psychosocial variable for the demographic variables that were significant. We also examined the unadjusted associations of the individual ITBS items with non-adherence (defined by the ITAS). We did not include the ITBS scores or individual ITBS items in adjusted, 
multivariate models, given that these barriers to adherence are part of the causal pathway leading to non-adherence and do not function as confounders.

For continuous variables, linearity in the logit was confirmed. Goodness of fit of the multivariate logistic regression models was assessed using the Hosmer-Lemeshow test [22]. Two-sided P-values $<0.05$ were considered statistically significant.

\section{Results}

\section{Study sample characteristics}

From May through September 2011, we enrolled 252 kidney transplant recipients out of 603 screened (41.7\%). At least 136 patients (22.6\%) explicitly declined to participate, because of lack of interest or inability to complete the study instruments during their clinic visit. The remaining screened patients were not approached, due to clinical concerns (e.g. acute illness) at the time of the office visit. The characteristics of the study subjects are shown in Table 1. Participants had a median age of 54.7 years (IQR 44.6-62.9) and were a median of 2.9 years post-transplant (IQR 1.4-5.8). Over one-quarter (27.0\%) were Black, 28.2\% had an annual household income $<\$ 35,000$ (for comparison, median household income in the United States was $\$ 50,054$ in 2011 [23]), and $43.7 \%$ had private medical insurance. A high percentage of patients had a diagnosis of glomerular disease $(40.5 \%)$ or received a kidney transplant from a live donor (62.7\%). Median serum creatinine was $1.4 \mathrm{mg} / \mathrm{dL}$ (IQR 1.1-1.8).

\section{Self-reported adherence}

The majority of study participants reported excellent adherence (Figure 1). 59.1\% scored 12 out of 12, while $26.6 \%$ scored either a 10 or 11 . The remaining $14.3 \%$ scored between 2 and 9 on the 12-point ITAS.

\section{Psychosocial measurements}

The health literacy of our study population was high, with 97.6\% having adequate health literacy on the sTOFHLA. The median sTOFHLA score was 35 (IQR 34-36). Only 6 patients (2.4\%) had sTOFHLA scores of 22 or less, which suggest marginal or inadequate health literacy.

Most subjects reported lower anxiety and depression levels on the HADS. On the anxiety component of the HADS, 206 (81.8\%) scored 0 to 7, 30 (11.9\%) scored 8 to 10 , and $15(6.0 \%)$ scored 11 to 21 , with 1 (0.4\%) incomplete. The median anxiety score was 4 (IQR 2-7). On the depression component, $232(92.1 \%)$ scored 0 to 7, $12(4.8 \%)$ scored 8 to 10 , and 5 (2.0\%) scored 11 to 21 , with $3(1.2 \%)$ of responses incomplete and not scorable. The median depression score was 1 (IQR 1-3).

Interpersonal support was high, with a median ISEL-12 score of 45 (IQR 39-47). Perceived stress was variable, with a median PSS-4 score of 4 (IQR 1-7).

\section{Univariate and multivariate analysis of psychosocial factors associated with adherence}

We performed unadjusted binary logistic regression models to examine the associations of candidate variables with non-adherence, defined as an ITAS score of 9 or less (Table 2). In the univariate logistic regression, non-adherence was associated with increased depression (on the HADS) and increased perceived stress (on the PSS-4) as well as lower household income and lack of employment. Younger age and increased anxiety appeared were not significantly associated with non-adherence but were considered candidate variables for the multivariate models (given their $\mathrm{P}<0.20$ ). Interpersonal support, health literacy, and clinical factors were not associated with nonadherence.

In separate multivariate models, we adjusted each candidate psychosocial variable (anxiety, depression, and perceived stress) for candidate demographic variables with $\mathrm{P}<0.20$ in the unadjusted model (age, income, employment status). Therefore, we constructed three multivariable models. In these adjusted models, anxiety, depression, and perceived stress were no longer significantly associated with non-adherence (Table 2).

\section{Barriers to adherence}

The median ITBS score was 16 (IQR 13-20). Higher scores on the ITBS (corresponding to more self-described barriers to adherence) were significantly associated with lower scores on the ITAS $(\mathrm{P}<0.001)$.

Seven of the thirteen individual items in the ITBS were significantly associated with adherence on the ITAS (Tables 3 and 4). Patients with lower adherence on the ITAS were more likely to rate increased agreement with statements that they skip doses when they go out of town or when they feel depressed. Patients with lower adherence were more likely to rate increased agreement that they run out of medications, find it hard to remember to take their medications, miss doses due to perceived side effects, miss doses when out of their daily routine, and skip doses when short of money.

The remaining six items in the ITBS were not associated with adherence (Table 4). In particular, lack of knowledge about the benefits of transplant immunosuppression was not associated with adherence [as measured by the ITBS items "I get confused about how to take my immunosuppressant medication"; "I do not understand when to take my immunosuppressant medication(s)"; and "I sometimes skip doses of my immunosuppressant medication(s) when I feel good (or better)"].

\section{Discussion}

In this cross-sectional study of prevalent adult recipients of kidney transplants, most patients were very adherent with their medications, at least by self-report. A minority 
Table 1 Socio-demographic and clinical characteristics of study population, based upon score on the Immunosuppressive Therapy Adherence Scale (ITAS)

\begin{tabular}{|c|c|c|c|c|c|}
\hline Characteristic & $\begin{array}{l}\text { Overall } \\
(\mathrm{N}=252)\end{array}$ & $\begin{array}{l}\text { ITAS score } \leq 9 \\
(\mathrm{~N}=36)\end{array}$ & $\begin{array}{l}\text { ITAS score }=10-11 \\
(\mathrm{~N}=67)\end{array}$ & $\begin{array}{l}\text { ITAS score }=12 \\
(\mathrm{~N}=149)\end{array}$ & $P$ \\
\hline Median age in years (IQR) & $54.7(44.6-62.9)$ & $48.1(38.1-61.1)$ & $54.6(37.7-64.3)$ & $55.0(46.3-62.6)$ & 0.17 \\
\hline Median years since transplant (IQR) & $2.9(1.4-5.8)$ & $3.3(2.2-5.1)$ & $2.7(1.4-5.7)$ & $2.6(1.3-5.9)$ & 0.47 \\
\hline Male, n (\%) & $151(59.9 \%)$ & $22(61.1 \%)$ & $36(53.7 \%)$ & $93(62.4 \%)$ & 0.48 \\
\hline Female, n (\%) & $101(40.1 \%)$ & $14(38.9 \%)$ & $31(46.3 \%)$ & $56(37.6 \%)$ & \\
\hline Race/ethnicity, n (\%) & & & & & 0.79 \\
\hline White & $145(57.5 \%)$ & $20(55.6 \%)$ & $39(58.2 \%)$ & $86(57.7 \%)$ & \\
\hline Black & $68(27.0 \%)$ & $12(33.3 \%)$ & $16(23.9 \%)$ & $40(26.9 \%)$ & \\
\hline Asian & $14(5.6 \%)$ & $1(2.8 \%)$ & $6(9.0 \%)$ & $7(4.7 \%)$ & \\
\hline Hispanic & $25(9.9 \%)$ & $3(8.3 \%)$ & $6(9.0 \%)$ & $16(10.7 \%)$ & \\
\hline Etiology of kidney disease, n (\%) & & & & & 0.57 \\
\hline Diabetes mellitus & $42(16.7 \%)$ & $8(22.2 \%)$ & $11(16.4 \%)$ & $23(15.4 \%)$ & \\
\hline Hypertension & $65(25.8 \%)$ & $13(36.1 \%)$ & $16(23.9 \%)$ & $36(24.2 \%)$ & \\
\hline Glomerulonephritis & $102(40.5 \%)$ & $10(27.8 \%)$ & $27(40.3 \%)$ & $65(43.6 \%)$ & \\
\hline Other & $43(17.1 \%)$ & $5(13.9 \%)$ & $13(19.4 \%)$ & $25(16.8 \%)$ & \\
\hline Highest education level, n (\%) & & & & & 0.65 \\
\hline $11^{\text {th }}$ grade or below & $16(6.4 \%)$ & $3(8.3 \%)$ & $4(6.0 \%)$ & $9(6.0 \%)$ & \\
\hline High school graduate or GED & $50(19.8 \%)$ & $8(22.2 \%)$ & $9(13.4 \%)$ & $33(22.2 \%)$ & \\
\hline Some college & $95(37.7 \%)$ & $14(38.9 \%)$ & $25(37.3 \%)$ & $56(37.6 \%)$ & \\
\hline College graduate or above & $90(35.7 \%)$ & $11(30.6 \%)$ & $28(41.8 \%)$ & $51(34.2 \%)$ & \\
\hline Unknown & $1(0.4 \%)$ & 0 & $1(1.5 \%)$ & 0 & \\
\hline Marital status, n (\%) & & & & & 0.72 \\
\hline Married & $167(66.3 \%)$ & $22(61.1 \%)$ & $42(62.7 \%)$ & $103(69.1 \%)$ & \\
\hline Widowed, divorced, separated, or never married & $78(31.0 \%)$ & $13(36.1 \%)$ & $22(32.8 \%)$ & $43(28.9 \%)$ & \\
\hline No response & $7(2.8 \%)$ & $1(2.8 \%)$ & $3(4.5 \%)$ & $3(2.0 \%)$ & \\
\hline Annual household income, n (\%) & & & & & 0.51 \\
\hline $0-\$ 34,999$ & $71(28.2 \%)$ & $15(41.7 \%)$ & $17(25.4 \%)$ & $39(26.2 \%)$ & \\
\hline$\$ 35,000-\$ 74,999$ & $57(22.6 \%)$ & $8(22.2 \%)$ & $17(25.4 \%)$ & $32(21.5 \%)$ & \\
\hline$\$ 75,000$ and above & $97(38.5 \%)$ & $9(25.0 \%)$ & $27(40.3 \%)$ & $61(40.9 \%)$ & \\
\hline Unknown & $27(10.7 \%)$ & $4(11.1 \%)$ & $6(9.0 \%)$ & $17(11.4 \%)$ & \\
\hline Employment status, n (\%) & & & & & 0.05 \\
\hline Working full-time & $92(36.5 \%)$ & $8(22.2 \%)$ & $27(40.3 \%)$ & $57(38.3 \%)$ & \\
\hline Working part-time & $36(14.3 \%)$ & $4(11.1 \%)$ & $12(17.9 \%)$ & $20(13.4 \%)$ & \\
\hline Not working & $124(49.2 \%)$ & $24(66.7 \%)$ & $28(41.8 \%)$ & $72(48.3 \%)$ & \\
\hline Primary health insurance, $\mathrm{n}$ (\%) & & & & & 0.62 \\
\hline Private insurance & $110(43.7 \%)$ & $12(33.3 \%)$ & $34(50.8 \%)$ & $64(43.0 \%)$ & \\
\hline Medicare & $106(42.1 \%)$ & $16(44.4 \%)$ & $24(35.8 \%)$ & $66(44.3 \%)$ & \\
\hline Medicaid & $27(10.7 \%)$ & $7(19.4 \%)$ & $6(9.0 \%)$ & $14(9.4 \%)$ & \\
\hline Medicaid and medicare & $7(2.8 \%)$ & $1(2.8 \%)$ & $2(3.0 \%)$ & $4(2.7 \%)$ & \\
\hline Charity or self-pay & $2(0.8 \%)$ & 0 & $1(1.5 \%)$ & $1(0.7 \%)$ & \\
\hline \multicolumn{6}{|l|}{ Type of kidney transplant, n (\%) } \\
\hline Deceased donor transplant & $94(37.3 \%)$ & $11(30.6 \%)$ & $22(32.8 \%)$ & $61(40.9 \%)$ & 0.35 \\
\hline Live donor transplant & $158(62.7 \%)$ & $25(69.4 \%)$ & $45(67.2 \%)$ & $88(59.1 \%)$ & \\
\hline
\end{tabular}


Table 1 Socio-demographic and clinical characteristics of study population, based upon score on the Immunosuppressive Therapy Adherence Scale (ITAS) (Continued)

\begin{tabular}{|c|c|c|c|c|c|}
\hline On dialysis prior to transplant, $\mathrm{n}(\%)$ & $190(75.4 \%)$ & $28(77.8 \%)$ & $51(76.1 \%)$ & $111(74.5 \%)$ & 0.84 \\
\hline Required dialysis during first post-transplant week, n (\%) & $30(11.9 \%)$ & $5(13.9 \%)$ & $4(6.0 \%)$ & $21(14.1 \%)$ & 0.22 \\
\hline Acute rejection episodes since transplant, $\mathrm{n}(\%)$ & $25(9.9 \%)$ & $8(22.2 \%)$ & $7(10.5 \%)$ & $10(6.7 \%)$ & 0.02 \\
\hline \multicolumn{6}{|l|}{ Calcineurin immunosuppressant, n (\%) } \\
\hline Cyclosporine & $34(13.5 \%)$ & $6(16.7 \%)$ & $9(13.4 \%)$ & $19(12.8 \%)$ & 0.83 \\
\hline Tacrolimus & $217(86.1 \%)$ & $30(83.3 \%)$ & $58(86.6 \%)$ & $129 * 86.6 \%)$ & 0.87 \\
\hline Median serum creatinine, in mg/dL (IQR) & $1.4(1.1-1.8)$ & $1.59(1.3-2.1)$ & $1.35(1.03-1.69)$ & $1.38(1.11-1.79)$ & 0.06 \\
\hline
\end{tabular}

Abbreviation: IQR Interquartile range.

$P$-values were obtained from chi-square test for proportions and Kruskal-Wallis test for medians.

of patients reported non-adherent medication-taking behaviors. Non-adherence was associated with higher perceived stress, anxiety, and depression. In adjusted, multivariate models, however, we could no longer detect any associations between non-adherence and stress, anxiety, and depression. The presence of self-described barriers to adherence was associated with non-adherence. These barriers to adherence may serve as potential targets for future interventions designed to increase medication adherence.

Our results confirm that a notable minority of kidney transplant recipients are non-adherent with their prescribed medications. Clearly, our study patients had been adherent enough to maintain a functioning allograft for a median of three years. Nevertheless, over $40 \%$ of patients were non-adherent in some form, as measured by the ITAS, and $14.3 \%$ were especially non-adherent, with an ITAS score of 9 or below. Other studies of prevalent kidney transplant recipients have reported similar rates of non-adherence [13]. Such long-term non-adherence may be associated with antibody-mediated rejection and allograft loss [3]. At least two ongoing clinical trials are testing interventions designed to increase adherence among prevalent kidney transplant recipients [24,25].

Our results also suggest possible targets for future interventions intended to increase medication adherence. Several specific barriers were significantly associated with non-adherence and may be amenable to modification. For example, changes in the patient's routine, including travel, were associated with non-adherence; contingency plans may help patients remain adherent despite changes in daily routines [13]. Patients admitted to running out of their medications or simply forgetting to take them; reminder cues, systems, and alarms can prompt patients to take their medications and to refill their medication prescriptions. At least one ongoing trial is attempting to determine whether reminders can increase adherence among incident kidney transplant recipients [26]. Interventions that address these "practical" barriers to adherence may be effective in increasing adherence.

This study has several important limitations. First, we assessed medication adherence by self-report, using a single instrument (the ITAS). Other methods of measuring adherence include electronic monitoring [27-29],

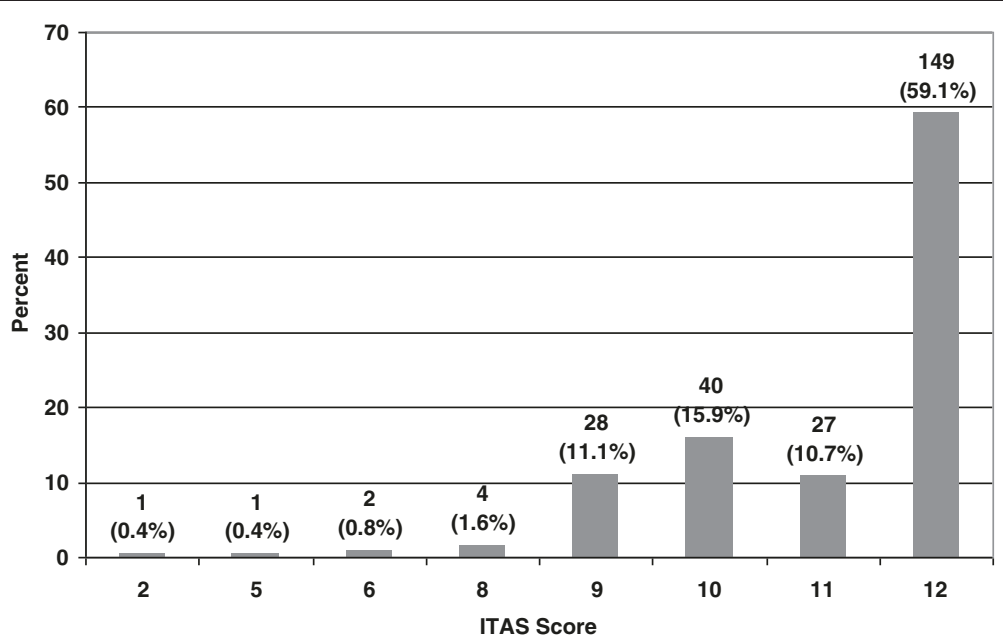

Figure 1 Distribution of scores on the Immunosuppressive Therapy Adherence Scale (ITAS). 
Table 2 Univariate and multivariate associations between Non-adherence (ITAS score of 9 or below) and psychosocial, socio-demographic, and clinical factors

\begin{tabular}{|c|c|c|c|c|c|c|}
\hline \multirow[t]{2}{*}{ Predictor } & \multicolumn{3}{|c|}{ Univariate models } & \multicolumn{3}{|c|}{ Adjusted, multivariate models* } \\
\hline & Odds ratio & 95\% confidence interval & $P$ & Odds ratio & $95 \%$ confidence interval & $P$ \\
\hline \multicolumn{7}{|l|}{ Psychosocial instruments } \\
\hline sTOFHLA Score & 1.10 & $0.93-1.30$ & 0.26 & & & \\
\hline HADS Anxiety Score & 1.10 & $1.00-1.22$ & 0.06 & 1.08 & $0.96-1.20$ & 0.21 \\
\hline HADS Depression Score & 1.14 & $1.02-1.28$ & 0.02 & 1.13 & $1.00-1.28$ & 0.06 \\
\hline ISEL-12 Score & 0.99 & $0.94-1.05$ & 0.71 & & & \\
\hline PSS-4 Score & 1.12 & $1.01-1.25$ & 0.04 & 1.10 & $0.97-1.24$ & 0.12 \\
\hline ITBS Score & 1.15 & $1.08-1.22$ & $<.001$ & & & \\
\hline \multicolumn{7}{|l|}{ Socio-demographic factors } \\
\hline Age (years) & 0.98 & $0.95-1.00$ & 0.09 & & & \\
\hline Female (vs. male) & 0.94 & $1.46-1.95$ & 0.87 & & & \\
\hline Non-white race (vs. white) & 1.10 & $0.54-2.24$ & 0.79 & & & \\
\hline $\begin{array}{l}\text { High school graduate and below } \\
\text { (vs. some college and above) }\end{array}$ & 1.28 & $0.59-2.77$ & 0.53 & & & \\
\hline Not married or partnered (vs. married or partnered) & 1.32 & $0.63-2.78$ & 0.47 & & & \\
\hline $\begin{array}{l}\text { Annual household income }<\$ 35,000 \\
\text { (vs. } \geq \$ 35,000 \text { ) }\end{array}$ & 2.16 & $1.01-4.62$ & 0.047 & & & \\
\hline Public health insurance (vs. private insurance) & 1.66 & $0.79-3.49$ & 0.18 & & & \\
\hline Unemployed (vs. employed) & 2.32 & $1.10-4.88$ & 0.03 & & & \\
\hline \multicolumn{7}{|l|}{ Clinical factors } \\
\hline Living donor (vs. deceased donor) & 1.42 & $0.66-3.03$ & 0.37 & & & \\
\hline \multicolumn{7}{|l|}{ Primary diagnosis (vs. glomerulonephritis) } \\
\hline Diabetes & 2.17 & $0.79-5.94$ & 0.13 & & & \\
\hline Hypertension & 2.30 & $0.94-5.61$ & 0.07 & & & \\
\hline Other & 1.21 & $0.39-3.78$ & 0.74 & & & \\
\hline Serum creatinine (mg/dL) & 1.83 & $1.18-2.83$ & 0.007 & & & \\
\hline Albumin (g/dL) & 0.83 & $0.33-2.05$ & 0.68 & & & \\
\hline $\begin{array}{l}6 \text { months to } 2 \text { years since transplant } \\
\text { (vs. }>2 \text { years since transplant) }\end{array}$ & 0.53 & $0.26-1.09$ & 0.08 & & & \\
\hline
\end{tabular}

${ }^{*}$ Adjusted for socio-demographic factors that had $\mathrm{P}<0.20$ (age, income, employment).

clinicians' collateral reports [30,31], serum assays for immunosuppressive medication concentrations [32-34], pill counts, and prescription refill and claims records [35]. Use of multiple methods, rather than a single method such as self-report, may be the most valid way to detect and measure non-adherence [36].

Second, we categorized adherence using cut-off scores for the ITAS. Although the ITAS itself has been validated, these cut-offs and categories of ITAS scores have not been validated for their association with outcomes. The clinical significance of different ITAS scores and categories is uncertain. To our knowledge, the correlation between specific amounts of adherence (whether measured on the ITAS or other instruments) and post-transplant outcomes remains unknown.
Second, we measured adherence among transplant recipients at a single transplant center in the northeastern United States. Our results are not necessarily generalizable to other transplant centers. Transplant centers differ in their staffing levels, the frequency with which they followup transplant recipients, the cultural competency of their providers, and the instructions they give transplant recipients regarding medications. Provider-level and health systems-level factors that vary between transplant centers may affect patients' adherence and contribute to intercenter variability in adherence [8].

Third, our convenience sample of prevalent transplant recipients who appeared for outpatient transplant follow-up was probably an especially adherent subset of kidney transplant recipients. Our study sample excluded 
Table 3 Significant barriers to immunosuppressant adherence, based upon responses to the ITBS (Immunosuppressive Therapy Barriers Scale)

\begin{tabular}{|c|c|c|c|c|c|}
\hline \multirow[t]{2}{*}{ ITBS questions } & $\begin{array}{l}\text { Overall } \\
(\mathrm{N}=252)\end{array}$ & $\begin{array}{c}\text { ITAS Score } \leq 9 \\
(\mathrm{~N}=36)\end{array}$ & $\begin{array}{l}\text { ITAS Score }=10-11 \\
(N=67)\end{array}$ & $\begin{array}{l}\text { ITAS Score =12 } \\
\quad(N=149)\end{array}$ & P-value \\
\hline & N (\%) & N (\%) & N (\%) & N (\%) & \\
\hline $\begin{array}{l}\text { I skip doses of my immunosuppressant } \\
\text { medication(s) when I go out of town }\end{array}$ & & & & & 0.04 \\
\hline Strongly disagree & $214(84.9)$ & $24(66.7)$ & $61(91.0)$ & 129 (86.6) & \\
\hline Disagree & $26(10.3)$ & $7(19.4)$ & $5(7.5)$ & $14(9.4)$ & \\
\hline Neutral & $5(2.0)$ & $2(5.6)$ & 0 & $3(2.0)$ & \\
\hline Agree & $4(1.6)$ & $2(5.6)$ & $1(1.5)$ & $1(0.7)$ & \\
\hline Strongly agree & $3(1.2)$ & $1(2.8)$ & 0 & $2(1.3)$ & \\
\hline $\begin{array}{l}\text { I miss doses of my immunosuppressant } \\
\text { medication(s) when I feel depressed }\end{array}$ & & & & & 0.009 \\
\hline Strongly disagree & $216(85.7)$ & $24(66.7)$ & 60(89.6) & 132 (88.6) & \\
\hline Disagree & $27(10.7)$ & $7(19.4)$ & $7(10.5)$ & $13(8.7)$ & \\
\hline Neutral & $4(1.6)$ & $2(5.6)$ & 0 & $2(1.3)$ & \\
\hline Agree & $4(1.6)$ & $3(8.3)$ & 0 & $1(0.7)$ & \\
\hline Strongly agree & $1(0.4)$ & 0 & 0 & $1(0.7)$ & \\
\hline $\begin{array}{l}\text { I often run out (or do not have enough) } \\
\text { of immunosuppressant medication(s) }\end{array}$ & & & & & $<0.0001$ \\
\hline Strongly disagree & $202(80.2)$ & $21(58.3)$ & 48 (71.6) & 133 (89.3) & \\
\hline Disagree & $30(11.9)$ & $5(13.9)$ & $12(17.9)$ & $13(8.7)$ & \\
\hline Neutral & $11(4.4)$ & $6(16.7)$ & $3(4.5)$ & $2(1.3)$ & \\
\hline Agree & $7(2.8)$ & $4(11.1)$ & $2(3.0)$ & $1(0.7)$ & \\
\hline Strongly agree & $1(0.4)$ & 0 & $1(1.5)$ & 0 & \\
\hline Missing & $1(0.4)$ & 0 & $1(1.5)$ & 0 & \\
\hline $\begin{array}{l}\text { It is hard for me to remember to take my } \\
\text { immunosuppressant medication(s) }\end{array}$ & & & & & $<0.0001$ \\
\hline Strongly disagree & 209 (82.9) & $24(66.7)$ & $50(74.6)$ & 135 (90.6) & \\
\hline Disagree & 35 (13.9) & $8(22.2)$ & $16(23.9)$ & $11(7.4)$ & \\
\hline Neutral & $5(2.0)$ & $2(5.6)$ & 0 & $3(2.0)$ & \\
\hline Agree & $2(0.8)$ & $2(5.6)$ & 0 & 0 & \\
\hline Strongly agree & 0 & 0 & 0 & 0 & \\
\hline Missing & $1(0.4)$ & 0 & $1(1.5)$ & 0 & \\
\hline $\begin{array}{l}\text { I miss a dose of my immunosuppressant } \\
\text { medication(s) when I think there may be side effects }\end{array}$ & & & & & 0.02 \\
\hline Strongly disagree & $215(85.3)$ & $26(72.2)$ & 56 (83.6) & 133 (89.3) & \\
\hline Disagree & $24(9.5)$ & $4(11.1)$ & $7(10.5)$ & $13(8.7)$ & \\
\hline Neutral & $5(2.0)$ & $2(5.6)$ & $1(1.5)$ & $2(1.3)$ & \\
\hline Agree & $7(2.8)$ & $4(11.1)$ & $2(3.0)$ & $1(0.7)$ & \\
\hline Strongly agree & 0 & 0 & 0 & 0 & \\
\hline Missing & $1(0.4)$ & 0 & $1(1.5)$ & 0 & \\
\hline $\begin{array}{l}\text { I miss doses of my immunosuppressant } \\
\text { medication(s) when I get out of my daily routine }\end{array}$ & & & & & $<0.0001$ \\
\hline Strongly disagree & $173(68.7)$ & $113(36.1)$ & $31(46.3)$ & 129 (86.6) & \\
\hline Disagree & 39 (15.5) & $9(25.0)$ & $13(19.4)$ & $17(11.4)$ & \\
\hline Neutral & $8(3.2)$ & $3(8.3)$ & $4(6.0)$ & $1(0.7)$ & \\
\hline
\end{tabular}


Table 3 Significant barriers to immunosuppressant adherence, based upon responses to the ITBS (Immunosuppressive Therapy Barriers Scale) (Continued)

\begin{tabular}{lcccc}
\hline Agree & $28(11.1)$ & $9(25.0)$ & $17(25.4)$ & $2(1.3)$ \\
$\quad$ Strongly agree & $3(1.2)$ & $1(2.8)$ & $2(3.0)$ & 0 \\
$\quad$ Missing & $1(0.4)$ & $1(2.8)$ & 0 & 0 \\
$\begin{array}{l}\text { I skip doses of my immunosuppressant } \\
\text { medication(s) when I am short of money }\end{array}$ & & & \\
$\quad$ Strongly disagree & $214(84.9)$ & $23(63.9)$ & $57(85.1)$ & $134(89.9)$ \\
Disagree & $29(11.5)$ & $7(19.4)$ & $9(13.4)$ & $13(8.7)$ \\
Neutral & $4(1.6)$ & $2(5.6)$ & $1(1.5)$ & $1(0.7)$ \\
Agree & $3(1.2)$ & $2(5.6)$ & 0 & $1(0.7)$ \\
Strongly agree & $2(0.8)$ & $2(5.6)$ & 0 & 0 \\
\hline
\end{tabular}

patients who no longer have a functioning allograft or follow-up with our transplant center. To qualify for the study, patients had to appear for a scheduled clinic appointment, which is itself a marker for adherence. Only 41.7\% of screened patients agreed to participate and complete our study questionnaires; we suspect that these study participants were more adherent than the overall population of screened patients. Our final study sample included large proportions of patients with favorable characteristics associated with increased allograft survival (e.g. recipients of live donor kidneys, patients with glomerular disease). Overall, this selection bias likely led us to overestimate the self-reported adherence of kidney transplant recipients in general.

Fourth, we were likely underpowered to detect associations between multiple factors and non-adherence. Although we examined over 250 transplant recipients, a study to examine the multiple factors plausibly associated with non-adherence may require a much larger study sample. Multi-center studies [6] may be necessary to accrue the larger numbers needed to properly study medication adherence.

Finally, we performed a cross-sectional study. This cross-sectional study design precluded meaningful analysis of the associations between adherence and acute rejection. For example, study participants with lower ITAS scores were more likely to have had prior rejection episodes. However, we lacked information on these patients' adherence and ITAS scores prior to the rejection episodes. An alternative study design would be a prospective cohort study, in which transplant recipients are followed over time. A cohort study would permit correlation of medication adherence with subsequent transplant outcomes, such as rejection or renal function.

\section{Conclusions}

In this single-center, cross-sectional study, a minority of recipients of kidney transplants was non-adherent with

Table 4 Unadjusted, univariate model of ITBS items associated with non-adherence (ITAS score of 9 or below)

\begin{tabular}{|c|c|c|c|}
\hline Immunosuppressant Therapy Barrier Scale (ITBS) items & Odds ratio & $95 \%$ confidence interval & $P$ \\
\hline I have to take the immunosuppressant medication(s) too many times per day. & 1.34 & $0.96-1.86$ & 0.09 \\
\hline I have to take too many capsules (or tablets) of my immunosuppressant medication(s) at one time. & 1.16 & $0.86-1.56$ & 0.34 \\
\hline I cannot tell if my immunosuppressant medication(s) is (are) helping me. & 1.18 & $0.86-1.62$ & 0.30 \\
\hline I skip doses of my immunosuppressant medication(s) when I go out of town. & 1.83 & $1.22-2.73$ & 0.003 \\
\hline I miss doses of my immunosuppressant medication(s) when I feel depressed. & 2.30 & $1.42-3.74$ & $<0.001$ \\
\hline I get confused about how to take my immunosuppressant medication(s). & 1.14 & $0.39-3.36$ & 0.81 \\
\hline I do not understand when to take my immunosuppressant medication(s). & 0.73 & $0.26-2.08$ & 0.55 \\
\hline I often run out (or do not have enough) of my immunosuppressant medication(s). & 2.22 & $1.50-3.30$ & $<0.001$ \\
\hline It is hard for me to remember to take my immunosuppressant medication(s). & 2.72 & $1.53-4.84$ & $<0.001$ \\
\hline I miss a dose of my immunosuppressant medication(s) when I think there may be side effects. & 2.05 & $1.32-3.19$ & 0.001 \\
\hline I sometimes skip doses of my immunosuppressant medication(s) when I feel good (or better). & 1.68 & $0.71-3.97$ & 0.24 \\
\hline I miss doses of my immunosuppressant medication(s) when I get out of my daily routine. & 1.78 & $1.35-2.37$ & $<0.001$ \\
\hline I skip doses of my immunosuppressant medication(s) when I am short of money. & 2.86 & $1.70-4.80$ & $<0.001$ \\
\hline
\end{tabular}

Odds ratios reflects odds of higher degree of agreement with each statement, based upon responses on a 5-point ordinal Likert scale (Strongly agree to strongly disagree). 
their medications. Non-adherence to medications was associated with increased anxiety, depression, and stress, but these associations did not persist in multivariate models. Practical barriers to adherence, such as forgetfulness and missing medications when one's routine is different, were significantly associated with non-adherence. These practical barriers may serve as promising targets for future interventions to increase adherence among recipients of kidney transplants.

\section{Competing interests}

This study was funded in part by Novartis Pharmaceuticals Corporation (East Hanover, New Jersey), as a research project with Drs. Weng and Demissie. Drs. Zacker is an employee of Novartis Pharmaceuticals. Dr. Chisholm-Burns receives licensing royalties from use of the ITAS and ITBS instruments from Pharmacotherapy Solutions.

\section{Authors' contributions}

FLW led the study conception and design; contributed to data acquisition, analysis, and interpretation; and drafted the manuscript. SC contributed to the study design and data acquisition, led the data analysis and interpretation, and contributed to revision of the manuscript. KMK contributed to the data analysis and interpretation and revision of the manuscript. CZ contributed to the study conception and design, data analysis and interpretation, and revision of the manuscript. MC-B contributed to the study design, data analysis and interpretation, and revision of the manuscript. KD contributed to the study conception and design, data analysis and interpretation, and revision of the manuscript. All authors read and approved the final manuscript.

\section{Acknowledgements}

This study was funded in part by Novartis Pharmaceuticals Corporation (East Hanover, New Jersey), as a research project with Drs. Weng and Demissie. Portions of this manuscript were presented at the American Transplant Congress in Boston, MA, on June 5, 2012.

\section{Author details}

'Saint Barnabas Medical Center, Renal \& Pancreas Transplant Division, 94 Old Short Hills Road, East Wing, Suite 305, Livingston, New Jersey 07039, USA. 2Department of Epidemiology, Rutgers School of Public Health, 683 Hoes Lane West, Piscataway, New Jersey 08854, USA. ${ }^{3}$ Novartis Pharmaceuticals Corporation, One Health Plaza, East Hanover, New Jersey 07936, USA. ${ }^{4}$ University of Tennessee Health Science Center, College of Pharmacy, 881 Madison Ave, Suite 264, Memphis, Tennessee 38163, USA.

Received: 7 March 2013 Accepted: 25 November 2013 Published: 1 December 2013

\section{References}

1. Butler JA, Roderick P, Mullee M, Mason JC, Peveler RC: Frequency and impact of nonadherence to immunosuppressants after renal transplantation: a systematic review. Transplantation 2004 77(5):769-776

2. Denhaerynck K, Dobbels F, Cleemput I, Desmyttere A, Schafer-Keller P, Schaub S, De Geest S: Prevalence, consequences, and determinants of nonadherence in adult renal transplant patients: a literature review. Transpl Int 2005, 18(10):1121-1133.

3. Sellares J, de Freitas DG, Mengel M, Reeve J, Einecke G, Sis B, Hidalgo LG, Famulski K, Matas A, Halloran PF: Understanding the causes of kidney transplant failure: the dominant role of antibody-mediated rejection and nonadherence. Am J Transplant 2012, 12(2):388-399.

4. Dew MA, DiMartini AF, De Vito Dabbs A, Myaskovsky L, Steel J, Unruh M, Switzer GE, Zomak R, Kormos RL, Greenhouse JB: Rates and risk factors for nonadherence to the medical regimen after adult solid organ transplantation. Transplantation 2007, 83(7):858-873.

5. Prendergast MB, Gaston RS: Optimizing medication adherence: an ongoing opportunity to improve outcomes after kidney transplantation. Clin J Am Soc Nephrol 2010, 5(7):1305-1311.
6. Greenstein S, Siegal B: Compliance and noncompliance in patients with a functioning renal transplant: a multicenter study. Transplantation 1998, 66(12):1718-1726.

7. Pinsky BW, Takemoto SK, Lentine $\mathrm{KL}$, Burroughs TE, Schnitzler MA, Salvalaggio PR: Transplant outcomes and economic costs associated with patient noncompliance to immunosuppression. Am J Transplant 2009, 9(11):2597-2606

8. Weng $\mathrm{FL}$, Israni AK, Joffe MM, Hoy T, Gaughan CA, Newman M, Abrams JD, Kamoun M, Rosas SE, Mange KC, et al: Race and electronically measured adherence to immunosuppressive medications after deceased donor renal transplantation. J Am Soc Nephrol 2005, 16(6):1839-1848.

9. Gordon EJ, Gallant M, Sehgal AR, Conti D, Siminoff LA: Medication-taking among adult renal transplant recipients: barriers and strategies. Transpl Int 2009, 22(5):534-545.

10. Evans RW, Applegate WH, Briscoe DM, Cohen DJ, Rorick CC, Murphy BT, Madsen JC: Cost-related immunosuppressive medication nonadherence among kidney transplant recipients. Clin J Am Soc Nephrol 2010, 5(12):2323-2328.

11. De Bleser L, Matteson M, Dobbels F, Russell C, De Geest S: Interventions to improve medication-adherence after transplantation: a systematic review. Transpl Int 2009, 22(8):780-797.

12. Chisholm MA, Lance CE, Williamson GM, Mulloy LL: Development and validation of the immunosuppressant therapy adherence instrument (ITAS). Patient Educ Couns 2005, 59(1):13-20.

13. Chisholm-Burns M, Pinsky B, Parker G, Johnson P, Arcona S, Buzinec P, Chakravati P, Good M, Cooper M: Factors related to immunosuppressant medication adherence in renal transplant recipients. Clin Transplant 2012, 26(5):706-713

14. Chisholm-Burns MA, Spivey CA, Wilks SE: Social support and immunosuppressant therapy adherence among adult renal transplant recipients. Clin Transplant 2010, 24(3):312-320.

15. Chisholm MA, Lance CE, Williamson GM, Mulloy LL: Development and validation of an immunosuppressant therapy adherence barrier instrument. Nephrol Dial Transplant 2005, 20(1):181-188.

16. Zigmond AS, Snaith RP: The hospital anxiety and depression scale. Acta Psychiatr Scand 1983, 67(6):361-370.

17. Cohen S, Hoberman HM: Positive events and social supports as buffers of life change stress. J App/ Soc Psychol 1983, 13(2):99-125.

18. Cohen S, Mermelstein R, Kamarck T, Hoberman HM: Measuring the functional components of social support. In Social support: Theory, research, and applications. Edited by Sarason IG, Sarason BR. The Hague, Holland: Martinus Nijhoff; 1985:73-94.

19. Cohen S, Kamarck T, Mermelstein R: A global measure of perceived stress. J Health Soc Behav 1983, 24(4):385-396.

20. Cohen S, Williamson GM: Perceived stress in a probability sample of the U.S. In The social psychology of health: Claremont Symposium on Applied Social Psychology. 1st edition. Edited by Spacapam S, Oskamp S. Newbury Park, CA: Sage; 1988:31-67.

21. Baker DW, Williams MV, Parker RM, Gazmararian JA, Nurss J: Development of a brief test to measure functional health literacy. Patient Educ Couns 1999, 38(1):33-42.

22. Hosmer DW, Lemeshow S: Applied logistic regression. 2nd edition. New York: John Wiley \& Sons, Inc.; 2000.

23. U.S. Census Bureau: Income, poverty, and health insurance coverage in the United States: 2011. U.S. Census Bureau: U.S. Department of Commerce, Economics and Statistics Administration; 2012.

24. Comparison of Medication Adherence Between Once and Twice Daily Tacrolimus in Stable Renal Transplant Recipients. [http://clinicaltrials.gov/ show/NCT01334333; NLM Identifier: NCT01334333]

25. Intervention to Improve Adherence in Teen Kidney Transplant (TAKE-IT). [http://clinicaltrials.gov/show/NCT01356277; NLM Identifier: NCT01356277]

26. Medication Adherence in Kidney Transplant Recipients Using Automated Reminders and Provider Notification. [http://clinicaltrials. gov/show/NCT01541384; NLM Identifier NCT01541384]

27. Nevins TE, Thomas W: Quantitative patterns of azathioprine adherence after renal transplantation. Transplantation 2009, 87(5):711-718.

28. Russell C, Conn V, Ashbaugh C, Madsen R, Wakefield M, Webb A, Coffey D, Peace L: Taking immunosuppressive medications effectively (TIMELink): a pilot randomized controlled trial in adult kidney transplant recipients. Clin Transplant 2011, 25(6):864-870. 
29. Russell $C L$, Cetingok M, Hamburger KQ, Owens S, Thompson D, Hathaway D, Winsett RP, Conn VS, Madsen R, Sitler L, et al: Medication adherence in older renal transplant recipients. Clin Nurs Res 2010, 19(2):95-112.

30. Schmid-Mohler G, Thut MP, Wuthrich RP, Denhaerynck K, De Geest S: Non-adherence to immunosuppressive medication in renal transplant recipients within the scope of the Integrative Model of Behavioral Prediction: a cross-sectional study. Clin Transplant 2010, 24(2):213-222.

31. Dharancy S, Giral M, Tetaz R, Fatras M, Dubel L, Pageaux GP: Adherence with immunosuppressive treatment after transplantation: results from the French trial PREDICT. Clin Transplant 2012, 26(3):E293-299.

32. Pai AL, Rausch J, Tackett A, Marsolo K, Drotar D, Goebel J: System for integrated adherence monitoring: real-time non-adherence risk assessment in pediatric kidney transplantation. Pediatr Transplant 2012, 16(4):329-334

33. Hsiau M, Fernandez HE, Gjertson D, Ettenger RB, Tsai EW: Monitoring nonadherence and acute rejection with variation in blood immunosuppressant levels in pediatric renal transplantation. Transplantation 2011, 92(8):918-922.

34. Shemesh E, Fine RN: Is calculating the standard deviation of tacrolimus blood levels the new gold standard for evaluating non-adherence to medications in transplant recipients? Pediatr Transplant 2010, 14(8):940-943.

35. Takemoto SK, Pinsky BW, Schnitzler MA, Lentine KL, Willoughby LM, Burroughs TE, Bunnapradist S: A retrospective analysis of immunosuppression compliance, dose reduction and discontinuation in kidney transplant recipients. Am J Transplant 2007, 7(12):2704-2711.

36. Schafer-Keller P, Steiger J, Bock A, Denhaerynck K, De Geest S: Diagnostic accuracy of measurement methods to assess non-adherence to immunosuppressive drugs in kidney transplant recipients. Am J Transplant 2008, 8(3):616-626.

doi:10.1186/1471-2369-14-261

Cite this article as: Weng et al: Prevalence and correlates of medication non-adherence among kidney transplant recipients more than 6 months post-transplant: a cross-sectional study. BMC Nephrology 2013 14:261.

\section{Submit your next manuscript to BioMed Central and take full advantage of:}

- Convenient online submission

- Thorough peer review

- No space constraints or color figure charges

- Immediate publication on acceptance

- Inclusion in PubMed, CAS, Scopus and Google Scholar

- Research which is freely available for redistribution 